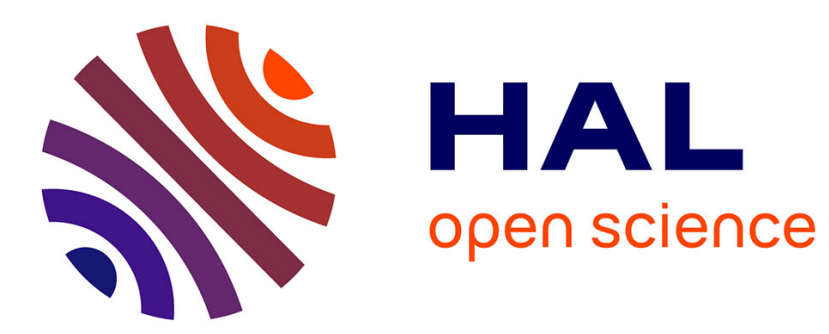

\title{
Clichés canoniques bouddhiques dans les légendes sur les débuts du bouddhisme au Japon
}

Hubert Durt

\section{To cite this version:}

Hubert Durt. Clichés canoniques bouddhiques dans les légendes sur les débuts du bouddhisme au Japon. Cahiers d'Extrême-Asie, 1985, no. 1, p. 11-20. 10.3406/asie.1985.856 . halshs-03134162

\section{HAL Id: halshs-03134162 \\ https://shs.hal.science/halshs-03134162}

Submitted on 8 Feb 2021

HAL is a multi-disciplinary open access archive for the deposit and dissemination of scientific research documents, whether they are published or not. The documents may come from teaching and research institutions in France or abroad, or from public or private research centers.
L'archive ouverte pluridisciplinaire HAL, est destinée au dépôt et à la diffusion de documents scientifiques de niveau recherche, publiés ou non, émanant des établissements d'enseignement et de recherche français ou étrangers, des laboratoires publics ou privés. 


\section{Clichés canoniques bouddhiques dans les légendes sur les débuts} du bouddhisme au Japon

\section{Hubert Durt}

\section{Citer ce document / Cite this document :}

Durt Hubert. Clichés canoniques bouddhiques dans les légendes sur les débuts du bouddhisme au Japon. In: Cahiers d'Extrême-Asie, vol. 1, 1985. pp. 11-20;

doi : https://doi.org/10.3406/asie.1985.856

https://www.persee.fr/doc/asie_0766-1177_1985_num_1_1_856

Fichier pdf généré le 06/02/2019 


\title{
CLICHÉS CANONIQUES BOUDDHIQUES
}

\author{
DANS LES LÉGENDES \\ SUR LES DÉBUTS DU BOUDDHISME AU JAPON*
}

\author{
HUBERT DURT
}

Je souhaiterais présentcr ici quclques résultats d'une étude menée avec des étudiants de l'Université d'Osaka sur certains des plus anciens textes racontant l'introduction du bouddhisme au Japon. Nos lectures de textes furent faites dans un esprit moins historique que-si l'on peut dire-littéraire: nous étions surtout à l'affût de traces, dans ces textes, d'influence des écritures bouddhiques.

Avant tout, il nous fallait nous demander si une telle lecture "littéraire" était légitime. La meilleure manière de s'en assurer était de comparer les récits japonais avec des récits sur l'adoption du bouddhisme dans d'autres régions. Il en existe un grand nombre et, pour chaque pays, les versions sont loin d'être uniformes. Le récit le plus élaboré est peut-être la légende de l'introduction du bouddhisme à Sri Lanka. Sa version la plus connue est celle, versifiée, du $M a-$ hāvamsa, ${ }^{1}$ une histoire nationale composée par un moine bouddhique, Mahānāma. Le Mahāvamsa se présente à nous avec des caractéristiques d'ouvrage d'édification et d'ouvrage ecclésiastique. Sans nous étendre sur les raisons de ce fait, bornons-nous ici à remarquer que l'influence de la communauté bouddhique (Samgha) est évidente sur cette sorte d'épopée qu'est le Mahāvamsa. De telles œuvres narratives, produites par le Saṃgha et centrées sur le Samgha, incluant en outre une histoire du bouddhisme depuis son origine, semblent être monnaie courante dans l'historiographie traditionnelle en Asie du Sud-Est.

Quoique dans mon séminaire à l'Université d'Osaka, il ait fallu se limiter à des textes écrits en chinois, il fut possible de ne pas laisser l'Asie du Sud-Est complètement en dehors du tableau. En effet, comme nous allons le voir, il existe dans le Canon bouddhique chinois (dans l'édition "Taishō Shinshū Daizōkyō" abrégée dorénavant en $\mathrm{T}$.) une version en chinois de la conversion au bouddhisme de l'Ile de Lañka.

Le temps ne nous a pas permis d'introduire dans notre enquête des textes concernant l'introduction du bouddhisme en Asie centrale quoique le Canon bouddhique chinois ne soit pas dépourvu de données à ce sujet. Qu'on se reporte, par exemple, au texte sur la conversion du Khotan ( $\mathrm{Li}$-yul), mieux connue dans sa version, d'ailleurs plus développée, en tibétain. ${ }^{2}$

La première année du séminaire à Osaka fut consacrée à une étude comparée

* Version révisée d'une communication faite à la $5^{e}$ conférence de l'Association internationale d'étude's bouddhiques à Oxford, le 21 août 1982 .

1) Mahāvamsa, chap. XIV, éd. par W. Geiger, Pāli Text Society, London, 1908; trad. par W. Geiger et M.H. Bode, The Mahävamsa or the Great Chronicle of Ceylon, nouvelle édition avec addendum par G.C. Mendis, P.T.S., 1964.

2) Voir R.E. Emmerick, Tibetan Texts concerning Khotan, London Oriental Series 19, London, 1969, et son appendice traitant de T. 2090 par E.N. Pulleyblank. 
de textes relatant en chinois des épisodes de la conversion de Sri Lanka, de la Chine, de la Corée et du Japon. La deuxième année fut plus spécifiquement consacrée au Japon: à l'histoire des événements des premières décades de l'adoption du bouddhisme dans ce pays et à l'évolution du traitement historiographique. ancien de ces événements.

Les textes qui firent l'objet de notre étude comparative sont les suivants:

I. L'histoire de la conversion de Lanka, telle qu'elle apparaît dans la traduction chinoise (T. 1462) de la Samantapāsādikā, commentairc du Vinaya pāli. ${ }^{3}$ Ce récit repose sur la même tradition que le récit, mentionné plus haut, du Mahãvamsa.

II. L'histoire de l'introduction du bouddhisme en Chine, que nous avons examinée à la lumière de l'étude classique d'Henri Maspero sur le sujet. ${ }^{4}$

III. L'histoire de l'adoption du bouddhisme dans les Trois Royaumes de Corée, spécialement dans le Royaume de Silla. Nous avons utilisé la compilation tardive (XIII ${ }^{\mathrm{e}}$ siècle) du Samguk rusa (T. 2039) ${ }^{5}$ pour la comparer avec les données utiles du recueil des "Vies des moines éminents de Corée" (T. 2063), traduit et annoté par Peter Lee. 6

Enfin, nous sommes arrivés aux récits japonais sur la pénétration du bouddhisme dans leur propre pays. La source principale, le Nihon Shoki 日本書紀 ou $\mathcal{N}$ ihongi 日本紀, ${ }^{7}$ est bien connue en Occident grâce aux traductions de Florenz ${ }^{8}$ et d'Aston. ${ }^{9}$ C'est une histoire nationale à structure annalistique comme les Histoires dynastiques chinoises.

Nous avons examiné aussi quelques chroniques ecclésiastiques au caractère plus bouddhique que le Nihon Shoki: I. le Jōgu Shōtoku Hōō Teisetsu 上宮聖德法王 帝說 (abrégé dans la suite en Teisetsu), ${ }^{10}$ une sorte d'aide-mémoire centré sur le Régent Shōtoku Taishi 聖德太子 (574-622), le personnage qui fut considéré comme le fondateur du bouddhisme japonais; et II., le Shōtoku Taishi Denryaku 聖德太子傳 歷 (abrégé dans la suite en Denryaku), ${ }^{11}$ une biographie populaire et très détaillée

3) Chapitre d'introduction (序品) du Chan kien liu pi-po-sha 善見律昆婆少 T.XXIV 1462 in 686c18III 689b22 correspondant au Bāhira nidāna vannanāa de la Samantapāsādikāa, éd. par J. Takakusu, M. Nagai, P.T.S. 1924, vol. I, p. 71.4-p. 82.4. Traductions du pāli: N.A. Jayawickrama, The Inception of Discipline and the Vinaya Nidāna, Sacred Books of the Buddhists, London, 1962, pp. 63-72; et du chinois: P.V. Bapat, A. Hirakawa, Bhandarkar Oriental Series 10, Poona, 1970, pp. 50-60.

4) "Le songe et l'ambassade de l'empereur Ming: Etude critique des sources", Bulletin de l'Ecole française d'Extrême-Orient X, 1910, pp. 95-130. Voir aussi E. Zürcher, The Buddhist Conquest of China, Leiden, 1959, vol. I, p. 20.

5) Extrait d'un texte perdu: Silla Pongi 新羅本記, T. XLIX 2039 In 987b2-c20.

6) Extr. de la biographie du moine Sŏk Pŏp-kong 濢法空, i.e. le roi Pŏp-hūng 法與 (T. L 2065 I 1018c7-1019a25). Traduction: Lives of Eminent Korean Monks, The Haedong Kosüng chŏn, HarvardYenching Institute Studies XXV, 1959, pp. 58-61.

7) Nous utilisions l'édition commentée de la collection Iwanami Koten Bungaku Taikei 岩波古典文叟 大系, vol. 68, Tokyð, 1965.

8) "Japanische Annalen A.D. 592-697 = Nihongi, Buch XXII - XXX", Mitteilungen der deutschen Gessellschaft für Natur- und Völkerkunde Ostasiens, Tokyס, 1903.

9) Nihongi, Chronicles of Japan from the Earliest Times to A.D. 697, publié en 1896; réimprimé, Rutland et Tokyo, 1972.

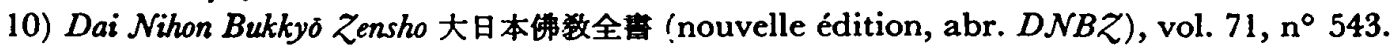

11) $D \mathcal{N B Z}$, vol. $71, \mathrm{n}^{\circ} 546$. 
du Régent Shōtoku. Les deux ouvrages subirent l'influence du Nihon Shoki, mais le Teisetsu, composé au Monastère Hōryūji 法隆寺 des environs de Nara, comprend également des éléments archaiques antérieurs à ceux provenant du Nihon Shoki.

Je répète que notre ambition n'était pas du tout une tentative de reconstruction historique des événements. Nous souhaitions seulement détecter les influences bouddhiques dans tous ces récits. Il pouvait s'agir de référence à des points de doctrine aussi bien que d'apparition de thèmes mythologiques ou hagiographiques.

Dans le domaine japonais, où notre étude fut la plus détaillée, il fut souvent possible de distinguer $1^{\circ}$ un canevas historique ou historiographique, $2^{\circ}$ des clichés apparemment d'origine japonaise, $3^{\circ}$ des stéréotypes bouddhiques, qui apparaissaient sous deux formes: a. des citations, parfois textuelles, de sütra, avec ou sans indication de source; b. des épisodes semblant empruntés à des écrits bouddhiques.

Avant d'envisager ces récits japonais, deux observations générales doivent encore être faites: d'abord, le lecteur ne doit pas s'étonner de voir ces récits traiter presque exclusivement de figures haut-placées comme des rois, empereurs et tout particulièrement un prince régent. Une donnée commune à tous les pays pourvus d'une tradition historique nationale est l'élaboration d'une version officielle des événements qui conduisirent à l'adoption du bouddhisme. Ces versions officielles présentent toujours l'introduction du bouddhisme comme prenant son départ au sommet de la hiérarchie sociale: par les bons offices du roi ou de l'empereur. C'est le cas dans le récit de la rencontre entre Mahinda, prince devenu religieux, fils du roi Asoka, et Devānampiya Tissa, roi de Lankka, comme c'est aussi le cas dans les évocations du rêve de l'empereur Ming 明 de la dynastie Han 漢 en Chine. Dans les relations de l'auto-sacrifice d'Ichadon 異次頓 en faveur du roi Pŏp-hŭng 法興 de Silla et dans les relations, au Japon, des échecs et succès du bouddhisme à la cour avant et durant la régence du prince Shōtoku, nous retrouvons ce même schéma. Au Japon, il existe aussi une discrète tradition ${ }^{12}$ concernant des cultes bouddhiques importés par des immigrants du continent avant leur introduction “officielle” par l'ambassade envoyée par Sŏng Myŏng 聖明, souverain du royaume coréen de Paekche 百斎. Cette tradition n'a heureusement pas été détruite, mais seulement recouverte par la version officielle des événements.

Une deuxième observation préliminaire va répéter ce qui a déjà été dit à propos de l'Asie du Sud-Est. Dans la plupart des cas, les auteurs de récits officiels sur l'introduction du bouddhisme étaient des moines bouddhiques. Même si en Chine, en Corée, au Japon, il n'y a pas de versions officielles codifiées par le Samgha, comme ce semble avoir été le cas en Asie du Sud-Est, il n'est pas surprenant que, vu la qualité de leurs auteurs, ces récits d'Extrême-Orient aient

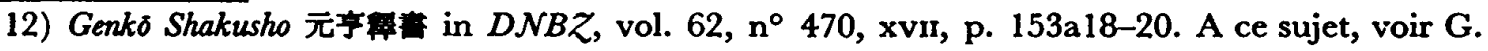
Renondeau, "La date de l'introduction du bouddhisme au Japon", T'oung Pao XIVIII, 1-2, 1959, p. 17-19; et J. Kamstra, Encounter or Syncretism, The Initial Growth of Japanese Buddhism, Leiden, 1967 , p. 246 sq. 
subi la marque de motifs littéraires dérivés de la littérature bouddhique.

La légende officielle racontant l'introduction du bouddhisme au Japon commence avec l'acceptation sous condition de la religion étrangère par un empereur présenté comme étant Shikishima 志發嶋, alias Kinmei 欽明, à une date controversée: 538 (12 jour du $10^{\mathrm{e}}$ mois de l'année tsuchinoe-uma 戊午 de Shikishima) dans le Teisetsu (III, 2) ou 552 ( $10^{\mathrm{e}}$ mois de la $13^{\mathrm{e}}$ année de Kinmei) dans le Nihon Shoki.

L'histoire de cette acceptation sous condition est l'objet d'une notice exceptionnellement longue dans le $\mathcal{N}$ ihon Shoki. Le message du roi de Paekche à Kinmei contient une mention de la diffusion du bouddhisme dans la direction de l'est, qui est la reprise d'un locus classicus des sütras de Prajñā-pāramita..$^{13}$ Cette mention figurait déjà dans la relation sur la diffusion du bouddhisme en Chine et a été reprise, avec référence à l'empereur Han Ming-ti 漢明帝, dans la relation sur le roi Pŏp-hŭng de Silla. ${ }^{14}$

Dans le message du roi de Paekche, le panégyrique du bouddhisme contient en outre des citations presque textuelles de la traduction du Suvarna-prabhāsasütra par Yi-tsing 義㐿. ${ }^{15}$ Cet emprunt, non déclaré mais significatif, avait déjà été remarqué par les premiers scoliastes modernes du Nihon Shoki. ${ }^{16}$ Récemment, dans l'histoire du bouddhisme de [l'époque] Asuka (Asuka Bukkyō shi kenkyū 飛鳥佛呚史研究), ${ }^{17}$ TAMURA Enchō a signalé, en relation avec cet emprunt, une influence possible du moine Dōji 道慈 (675-744). Dōji est surtout connu comme membre de l'équipe de rédaction du Nihon Shoki publié en 720 . Avant cette date, Dōji avait séjourné dix-sept ans, de 702 a 718, en Chine. A Tch'ang-ngan, il résidait au fameux monastère Si Ming sseu 西明寺, le centre de traduction où Yi-tsing a justement réalisé en 703 sa traduction du Suvarna-prabhāsa-sūtra.

Nous pouvons déceler un nouveau cas de fabrication littéraire dans l'évocation de la persécution du bouddhisme qui conclut la longue notice de 552 dans le Nihon Shoki. Cette évocation semble un doublet anticipant des événements décrits sous trois entrées de l'année 585 dans le même Nihon Shoki: celles du $24^{\mathrm{e}}$ jour du $2^{\mathrm{e}}$ mois et des $1^{\mathrm{er}}$ et $30^{\mathrm{e}}$ jours du $3^{\mathrm{e}}$ mois durant la $14^{\mathrm{e}}$ année du règne du successeur de Kinmei, l'empereur Bidatsu 敏達.

Nous voyons donc que la fameuse notice de $552 \mathrm{du}$ Nihon Shoki sur la première acceptation du bouddhisme semble bien être une assez douteuse combinaison d'événements anti-datés (552 par rapport à 585) et post-datés (552 par rapport à 538) liés ensemble par des rappels, reconnus ou camouflés, de sūtras bouddhiques. A propos de la date de 552 , Tamura ${ }^{18}$ a en outre émis l'hypothèse que cette an-

13) Voir E. Lamotte, Trailé de la Grande Vertu de Sagesse, vol. I, Louvain, 1944, p. 25-26; Yamada

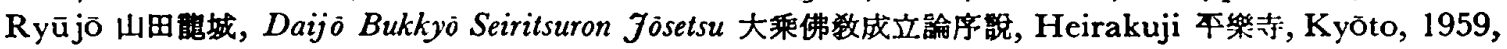
pp. 523-532.

14) T. XLIX 2039 II $987 b 10$.

15) T. XVI 665 I 406a17-20, vi 432b12-13, III 413a26, x 455a16-17. Traduit par J. Nobel, Leiden, 1958.

16) Voir Renondeau, art. cit., p. 21.

17) Hanawa Shobo 塙数, Tōkyర, 1969, p. 168-177.

18) Op. cit., p. 172-173. 
née a été choisie, peut-être par Dōji, afin de coincider avec l'une des spéculations chinoises sur l'année inaugurale de la période de déclin de la Loi (mappō 末法), un thème remontant à la prédication ancienne du Buddha. ${ }^{19}$

Ensuite, il semble que le sort du bouddhisme au Japon a été fixé par une bataille qui eut lieu en 587 ( $7^{\mathrm{e}}$ mois de la $2^{\mathrm{e}}$ année du règne de Yomei 用明, successeur de Bidatsu; en fait, déjà sous le règne du successeur de Yomei: l'empereur Sujun 崇峻). Dans le Nihon Shoki, la narration de la victoire du clan probouddhique Soga 蘇我 sur le clan anti-bouddhique Mononobe 物部 eșt axée sur la légende d'une intervention, quelque peu anachronique, du prince impérial destiné à devenir sous le nom de Shōtoku le symbole national et œcuménique de l'acceptation au Japon du bouddhisme et de la civilisation chinoise. Dans cette narration, nous trouvons à nouveau une référence au Suvarna-prabhāsasūtra dans la traduction de Yi-tsing. ${ }^{20}$

Remarquons ici qu'avant l'introduction de cette traduction de Yi-tsing, relativement tardive, d'autres traductions de ce sütra étaient déjà connues au Japon. Il semble qu'il s'agissait même d'un des sūtras Mahāyāniques les plus populaires. ${ }^{21}$ Il était spécialement fameux pour sa propagation du culte des quatre dieux-rois gardiens. ${ }^{22}$ En dehors des témoignages littéraires, la popularité de ce sütra se manifeste aussi dans les oeuvres d'art: plusieurs statues des quatre dieux gardiens remontant à la période Asuka (552-645) et la célèbre illustration d'un $\mathcal{F} a \overline{t a k a^{23}}$ narré dans ce sütra que l'on voit peinte sur un panneau de l'autel Tamamushi Zushi 玉蟲倣子 du Hōryūji.

Plusieurs esquisses biographiques anciennes sur Shōtoku Taishi ont récemment fait l'objet de monographies spécialisées. Pour n'en mentionner que quelquesunes, citons l'édition annotée du Teisetsu par IEnAGA Saburō 家永三郎24 et le travail sur le Hoketsuki 蒱闑記 par SHINKAWA Tokio 新川登男.25 Nous manquons cependant encore d'un examen général exhaustif de toute l'historiographie concernant Shōtoku. Il est intéressant de noter que la tentative la plus détaillée poussée dans cette direction a été faite, il y a quarante ans, non en japonais mais en allemand. L'ouvrage encyclopédique, généralement inconnu, "Shōtoku Taishi" par Hermann Bohner, ${ }^{26}$ quoique insuffisamment critique selon les normes d'aujourd'hui, reste très utile et est injustement négligé.

19) E. Lamotte, "Prophéties relatives à la disparition de la Bonne Loi", in R. de Berval éd., Présence du Bouddhisme, Saigon, 1959, pp. 657-663. Voir aussi E. Lamotte, Histoire du bouddhisme indien, Louvain, 1958, pp. 210-222.

20) T. XVI 665 x 451 b10. Cf. aussi Tamura Enchö, "Early Buddhism in Japan", in Bulletin of the Faculty of Literature, Kyushu University, XIII, 1971, p. 30-31.

21) Cr. Takeda Choten 竹用輰典, “Kodai Nihon ni okeru Bukkyo kyठten no juyõ" 古代日本に おけける仏教経典の受容, Taishō Daigaku Kenkyū kiyō 63, 1977, pp. 1-23.

22) Son chapitre VII est consacré aux Calur-mahāāajjā.

23) Le Jātaka du Prince Mahāsattva dans T. XVI 663 (trad. de Dharmakșema) Iv 354a19356c18. Voir aussi E. Lamotte, Traité, I, p. 143, note 1.

24) Jógū Shötoku Hōo Teisetsu no kenkyū 上宫嫼德法王帝說の研究, Sanseido 三省堂, Tōkyō, 1949.

25) Jojgū Shôtoku Taishi Den Hoketsuki no kenkyū 上宮聖德太子傳補関記の研究, Yoshikawa Köbunkan 吉川弘文館, Tokyo, 1980.

26) Deutsche Gesellschaft für Natur- und Völkerkunde Ostasiens, Tökyo, 1940. 
Parmi les nombreux problèmes auxquels est confronté le chercheur étudiant ces biographies anciennes, nous mentionnerons ici ceux qui concernent les fragments de traditions autonomes de temples ou monastères. Le bouddhisme japonais primitif était étroitement lié aux destinées de quelques monastères illustres. Comme l'a montré récemment Hayashi Mikiya 林幹雨,27 on peut distinguer, parmi les sources sur Shōtoku Taishi, celles qui sont liées à la tradition du Shitennōji 四天王寺, “monastère des quatre dieux-rois [gardiens]", dans la ville contemporaine d'Osaka, et les sources qui sont liées au Höryūj: "monastère de la prospérité de la Loi", dans le voisinage de Nara. Un troisième monastère, le Hōkōji 法興寺, monastère du triomphe de la Loi, qui est à présent appelé Asukadera 飛鳥寺 dans le village contemporain d'Asuka, semble avoir joué un rôle important du vivant de Shōtoku Taishi et durant quelques décades après sa mort.

La destinée du Hōkōji fut inséparable des vicissitudes du clan Soga. Il est possible qu'on trouve un témoignage de son importance dans la trace, dans une inscription de 623 commentée dans le Teisetsu III, 2, 3, de l'usage d'un nom d'ère bouddhique qui semble avoir été usité au moins jusqu'à la mort de Shōtoku en 622. Cette ère porte le nom de "triomphe de la Loi" (Hōkō 法興); peut-être est-il dérivé du nom du monastère Hōkōji. "Triomphe de la Loi" était un nom auspicieux et significatif en ces siècles. Nous avons déjà rencontré Pŏp-hŭng, la prononciation coréenne de ce terme, qui était le nom posthume du roi de Silla qui adopta le bouddhisme sous son règne (514-540).

Comme Claude Maître ${ }^{28}$ l'a déjà indiqué, 591, la $1^{\text {re }}$ année de l'ère japonaise "triomphe de la Loi", ne correspond à aucun événement bien connu de l'histoire du bouddhisme japonais, mais le même Claude Maître, dans un ouvrage plus ancien, ${ }^{29}$ nous avait mis en garde contre une trop grande confiance en la datation du Nihon Shoki jusqu'à la fin du règne du successeur de Sujun, l'impératrice Suiko 推古 (593-628). La relation entre ère Hōkō et monastère Hōkō est loin d'être claire, mais on remarque que le Nihon Shoki date avec emphase le début du bouddhisme au Japon en 585 (9. mois de la 13 $1{ }^{\mathrm{e}}$ année de Bidatsu). Cet établissement du bouddhisme correspond, dans l'esprit du Nihon Shoki, à la découverte d'une relique miraculeuse (shari 舍利 $=$ sk. śarìra) et à son installation solennelle dans un sanctuaire du clan Soga. Ensuite, le Nihon Shoki mentionne le vœu de construire le Hōkōji pris par Soga Umako 蘇我馬子 après la bataille de 587 entre son clan et le clan Mononobe. Le Nihon Shoki donne encore des informations précises sur la construction de ce monastère. C'est en 593 ( $1^{\text {re }}$ année de Suiko) et non en 591 ( $1^{\text {re }}$ année de $H \bar{o} k \bar{o}$ ) que le Hōkōji est virtuellement consacré par le dépot d'un shari du Buddha dans la pierre de base du pilier axial du stüpa, appelé ici satsu 刹, du Hōkōji. Nous pouvons ajouter ici que cette discordance chronologique entre 591 et 593 n'est pas la seule dans les chroniques anciennes

27) Taishi shinkō no kenkyū 太子信仰の研究, Yoshikawa Kōbunkan, Tơkyō, 1980.

28) "Une inscription japonaise de l'an 623", Etudes Asiatiques, $25^{\circ}$ anniversaire de l'EFEO, Paris, 1925, pp. 402-432.

29) "La littérature historique du Japon, des origines aux Ashikaga", BEFEO III, 1903, p. 576580. Cf. aussi E. Gaspardone, "La chronologie ancienne du Japon", Journal Asiatique, avril-juin 1938, p. 235. 
traitant de Shōtoku Taishi.

L'adoption de Shōtoku Taishi dans la tradition historique des monastères les plus prestigieux du bouddhisme japonais ancien n'exclut pas des associations avec des temples moins fameux ou plus récents. On pourrait parler d'une convention dans la littérature des "fondations de temples et sanctuaires" (Jisha engi 寺社縁起) qui consistait à annexer l'illustre personnalité de Shōtoku dans les innombrables légendes de fondation. Il existe déjà des traces de cette tendance dans le Nihon Shoki.

A Shōtoku Taishi furent également attribués des commentaires sur trois sūtras: le Vimalakīrti-nirdeșa, le Saddharma-pundarika-sütra et la Ratnamālā-devi-pariprcchā. Avec le Suvarna-prabhāsa-sūtra déjà mentionné, ces trois textes semblent avoir été parmi les Mahäyanasūtras les mieux connus en Extrême-Orient durant la période de développement de l'érudition bouddhique, avant la vogue du Tantrisme. En Chine, le Vimalakîrti, le Saddharma et la Ratnamālā avaient déjà été l'objet de lecture publique (sin.-jap. $k \bar{o}$ 講) par le fameux empereur $\mathrm{Wu}$ 武 de la dynastie Leang 梁 qui régna de 502 à 549.30 La protection que cet empereur, inspiré par l'exemple du roi Aśoka, accorda au bouddhisme semble avoir influencé ses contemporains coréens, ${ }^{31}$ déjà mentionnés plus haut, le roi Sŏng Myŏng 聖明 de Paekche (règne 523-554) et le roi Pŏp-hŭng de Silla (règne 514-540), et, un demi-siècle après la mort de Leang $\mathrm{Wu}$ ti 梁武帝, le régent japonais Shōtoku.

L'idée traditionnelle selon laquelle les trois commentaires de sūtra furent composés dans l'entourage de Shōtoku Taishi n'est pas encore à exclure. L'adversaire le plus tenace de la théorie de leur attribution à Shōtoku, le Professeur FujIEDA Akira, a projeté de nouvelles lumières sur le caractère typiquement chinois du VI ${ }^{\mathrm{e}}$ siècle du commentaire de la Ratnamālā en le comparant avec un texte analogue provenant de Touen-houang. ${ }^{32}$ De ces commentaires, seul celui du Saddharma subsiste à l'état de manuscrit. Des autres, nous ne connaissons que des éditions imprimées tardives. Le contenu du commentaire du Saddharma est médiocre mais pourrait être assez archaĩque. Sa belle calligraphie, dont l'attribution à la main de Shōtoku Taishi est des plus douteuses, a valu au Régent la réputation de maître calligraphe. ${ }^{33}$

Le Teisetsu, II, 3 présente ces commentaires comme révélés à Shōtoku par un homme d'or qui lui serait apparu en rêve. Le rêve de l'homme d'or était déjà l'élément principal de la légende de l'attrait du bouddhisme sur l'empereur Ming dans l'historiographie chinoise. Une tradition tardive a identifié cet homme

30) Une bonne analyse chronologique des activités bouddhiques de Leang Wou-ti se trouve dans Mochizuki Bukkyó Daijiten 望月佛呚大典, Tōkyō, 1933, s.v. Butei (vol. V, p. 4481a-4482c.) et dans l'étude de Suwa Gijun 諏訪義純, “A Study of Liang Wu Ti's Buddhist Chronology”, Bukkyó Shigaku kenkyū 佛呚史學研究 26, 1 (1983; , p. 72-94 et 2 (1984), p. 45-76.

31) Sur les relations "bouddhiques" entre la dynastie des Leang et le royaume de Paekche, cf.

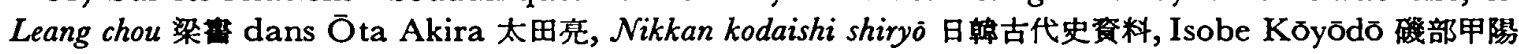
堂, Tokyo, 1927, p. 63; entre Leang et Silla, cf. T. XLIX 2039 mi 987c23.

32) Shōtoku Taishi shū 聖镘太子集, Nihon shisō taikei 日本思想大乐, vol. 2, Iwanami, Tōkyō, 1975.

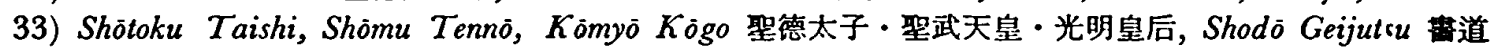
秐街 vol. II, Chūō kōron 中央公論, Tōkyō, 1976. 
d'or au bodhisattva Avalokiteśvara “Sauveur du monde" (Guse Kannon 救世觀 音). ${ }^{34}$ Une statue de Guse Kannon, de l'époque Asuka, est encore à présent le principal objet de culte (honzon 本夏) dans le Pavillon du rêve (Yume-dono 夢殿) au Hōryūji.

On attribue également à Shōtoku Taishi la paternité de la Constitution en XVII articles telle qu'elle est citée dans le Nihon Shoki en 604 ( $3^{\mathrm{e}}$ jour du $4^{\mathrm{e}}$ mois de la $12^{\mathrm{c}}$ année de Suiko). Dans ce texte moralisant, nous trouvons à côté de nombreuses références aux classiques chinois, une allusion assez inattendue aux quatre types de naissance (sk. yoni) comme nous les connaissons d'après les Ägamas bouddhiques. ${ }^{35}$

Déjà dans les entrées biographiques du Nihon Shoki à propos de Shōtoku Taishi, on peut discerner quelques éléments hagiographiques qui semblent des emprunts et des adaptations de l'hagiographie de Śăkyamuni. C'est le cas, comme nous le verrons plus loin, pour les circonstances de la naissance de Shötoku. Ici, nous mentionnerons sa rencontre avec un mendiant agonisant à Kataoka 片岡 en 613 ( $1^{\mathrm{er}}$ et $2^{\mathrm{e}}$ jour du $12^{\mathrm{e}}$ mois de la $21^{\mathrm{e}}$ année de Suiko). La similitude avec les quatre fameuses rencontres du Bodhisattva, le futur Śākyamuni, n'est que superficielle, mais le fait que le Régent donne son manteau au mendiant, puis, après sa mort, le reprenne pour s'en revêtir, nous rappelle non seulement l'échange de vêtements dans la légende de Śākyamuni, mais semble surtout une sorte d'adaptation japonaise du culte par vêtement $P$ ämsukülika. ${ }^{36}$ D'autre part, dans une entrée de 628 (la $36^{\mathrm{e}}$ année de Suiko, mais déjà sous le règne de son successeur, l'empereur Jomei 舒明), les derniers mots de Shōtoku Taishi sur son lit de mort sont rapportés sous la forme d'une ghāta, moins frappante, mais coulée dans le même moule que la derniére ghātā de Śākyamuni dans le plus courant des récits de son Parinirvāna. ${ }^{37}$

Comme il est habituel dans la littérature hagiographique, la biographie de Shōtoku Taishi se fit de plus en plus élaborée au fur et à mesure que le personnage historique s'éloignait dans le passé. Une représentation standard du personnage fut mise au point dans le Denryaku, une biographie composée au $\mathrm{X}^{\mathrm{e}}$ siècle par un membre de la famille Fujiwara 藤原. L'auteur se conforme à la structure annalistique du Nihon Shoki, mais fut aussi fort influencé par la tradition du Shitennōji et par la secte Tendai 天台.

Le Denryaku, qui devait exercer une grande influence, spécialement sur la riche iconographie de Shōtoku Taishi, emprunte beaucoup à beaucoup de sources. Il éclaire de nouveaux aspects de la légende de Houei-sseu 慧思 (515-577), le second patriarche de l'école T'ien-t'ai, récemment étudié d'après les sources chinoises par

34) Voir Mochizuki, Bukkyō daijiten, s.v. (vol. I, p. 677b-678a).

35) T. I 1 virr 50c8; T. II 125 xvIr 632a7-19; cf. E. Lamotte, Traité, I., p. 483, n. 2; Louis de la Vallée Poussin, Abhidharmakosa, réimpr. M.C.B. XVI, Bruxelles, 1970, III, p. 28.

36) A comparer avec Pañsutül au Cambodge, en Thailande et au Laos, cf. F. Biz.ot, Le don de soi-même, Recherches sur le bouddhisme khmer III, Publication de l'Ecole française d'Extrême-Orient, CXXX, Paris, 1981.

37) T. I. 1 iv $26 \mathrm{~b} 18$. 
Paul Magnin. ${ }^{38}$ La concordance chronologique entre la date de la mort de Houeisseu et celle de la naissance de Shōtoku semble avoir été utilisée dans un but de propagande par Sseu-to 思詫 (VIII'e siècle), l'un des premiers missionnaires T'ient'ai au Japon. Pour Sseu-tö, Shōtoku était une réincarnation de Houei-sseu. ${ }^{39}$

En outre, pour enrichir encore davantage la biographie de Shōtoku, le Denryaku, plus explicitement que le Nihon Shoki, semble avoir emprunté à la biographie d'un autre prince héritier, le futur Śākyamuni.

Considérons d'abord la naissance de Shōtoku. Nous trouvons dans le Denryaku, mais pas dans la légende plus ancienne de Shōtoku Taishi, un cas de docétisme bien connu dans la légende de Śākyamuni où il fut étudié par Alfred Foucher. ${ }^{40}$

Dans le Denryaku, la conception de Shōtoku, en 571 ( $^{\mathrm{er}}$ mois de la $32^{\mathrm{e}}$ année de Kinmei) est une sorte de parthénogénèse. Comme le roi Śuddhodana, le futur empereur Yomei accepte de perdre son rôle de père. Au lieu de l'éléphant du rêve de la reine Māyā, nous avons dans la légende japonaise un moine qui est seulement désigné par sa couleur: le "religieux à couleur d'or" (Konjikisō 金色挸). Nous avons déjà observé le leit-motiv de l'homme d'or en rapport avec le rêve de Han Ming-ti et avec l'inspiration des trois Commentaires de Shōtoku Taishi. Ce moine se présente à l'épouse du futur empereur Yomei comme un “bodhisattva sauveur du monde" (Guse Bosatsu 救世菩薩), une référence implicite, comme nous l'avons vu, à Avalokiteśvara. Il vient de la région de l'Ouest, ce qui est typiquement amidiste. Il déclare que, conformément à son vœu, il souhaite prendre résidence ( $j u k u$ 宿) en ses entrailles ( $f u k u$ 腹). Elle répond que ses entrailles sont souillées, mais néanmoins le moine se réduit à une taille minuscule et s'introduit dans la bouche de la princesse qui devient enceinte. Je ne connais pas d'autre récit analogue à cette étrange légende sur l'origine de Shōtoku, mais $\mathrm{S}$. Anderson a montré qu'une naissance miraculeuse, laissant à l'écart le père, est une donnée commune dans les légendes des saints personnages du Japon. ${ }^{41}$

La naissance elle-même se produit durant une sortie de la princesse, comme ce fut le cas de la reine Māyā. Suivant une explication bien établie, le nom du futur régent, Umayado 廐戶, vient de l'écurie (uma-yado 馬屋戶) en face de laquelle la princesse accoucha de Shōtoku.

Dans les récits de la jeunesse de Shōtoku, il y a à nouveau plusieurs ressemblances avec la biographie du futur Śākyamuni: excellence dans les études, dans les exercices physiques. Beaucoup de ces notations, en particulier, l'anecdote selon laquelle il se soumit de lui-même à une punition par son père, semblent être hagiographiques. Plus généralement, l'importance accordée à des épisodes de l'enfance de Shōtoku, comme aussi de l'enfance de plusieurs éminentes figures du Japon, et leur représentation en "[Saints] enfants" ( $d \bar{o} j i$ 童子) semblent être une caractéristique de la religiosité japonaise. Ce thème semble moins développé

38) La vie et l'oeuvre de Huisi 慧思 (515-577), Les origines de la secte bouddhique chinoise du Tiantai, PEFEO CXVI, Paris, 1979.

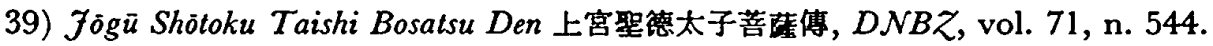

40) Vie du Buddha, Paris 1949, p. 32-44.

41) "Legends of Holy Men in Early Japan", Monumenta Serica XXVIII (1969), p. 293. 
dans le bouddhisme indien ou chinois. En outre, en ce qui concerne le terme "taishi" (太子), c.-à-d. "Prince héritier", qui est le titre le plus habituellement donné à Shōtoku, on notera que dans les textes bouddhiques, le terme sanskrit kumāra est traduit soit par dōji, c.-à-d. enfant, soit par taishi, prince héritier. ${ }^{42}$

Le fait que Shōtoku est resté un laìc et est devenu le prince héritier et le régent (kōtaishi 皇太子) pour sa tante, l'impératrice Suiko, qui lui survécut, montre que sa carrière suivit la voie du roi Cakravartin, non celle du Buddha. Néanmoins, l'histoire de sa vie contient encore de nombreux événements imprégnés de signification religieuse.

L'un de ces événements est le choc que subit Shōtoku quand il fut témoin du combat entre une biche et un chien (Denryaku, $3^{\mathrm{e}}$ mois de la $22^{\mathrm{e}}$ année de Suiko). Cela nous rappelle, même dans le choix des termes, le spectacle de petits animaux occupés à s'entretuer, spectacle qui, selon certaines traditions, emmena le futur Śākyamuni à sa première méditation sous l'arbre jambu. Shōtoku se réfère immédiatement à la doctrine bouddhique bien connue de la transmigration: dans une existence antérieure, ces animaux devaient être des êtres humains se haisssant mutuellement. Dans le cas de Śăkyamuni, une version seulement de cette légende lie le spectacle à la loi du karman. Ce lien dans l'esprit du Bodhisattva se rapporte au futur: ces massacres vont encore entraîner de plus désastreuses conséquences. C'est une raison supplémentaire pour quitter ce monde déprimant aussi rapidement que possible. ${ }^{43}$

On pourrait encore dire plus sur l'adaptation de thèmes de la légende du Buddha dans la biographie de figures nationales du Japon. Il n'est pas étonnant que quelques siècles plus tard l'"héroisation" de Shōtoku Taishi avait tellement bien réussi que la relation semble s'être inversée. Ce fut le futur Śākymuni qui fut représenté sous les traits du prince héritier Shōtoku dans un sūtra illustré du XIII ${ }^{\mathrm{e}}$ siècle, ${ }^{44}$ une nouvelle moûture du Kako genzai ingakyō emaki 過去現在因 果經繪危. Le texte de cet emaki, une vie de Śākyamuni (T. 189), proche du Buddhacarita et traduite en chinois au $V^{e}$ siècle, fut l'objet d'une illustration archaíque durant la période Nara 奈良 (VIII I siècle) et à nouveau d'une illustration modernisante, celle dont il est question ici, durant la période Kamakura 鎌倉 (11831331). Ce processus d'assimilation ne fut pas limité à Shōtoku Taishi. Une étude récente ${ }^{45}$ a montré que les représentations populaires de la mort des grandes figures du bouddhisme japonais, Hōnen 法然 et Nichiren 日莲, étaient souvent inspirées de la mise en scène du Parinirvạna du Buddha.

42) Cf. E. Zürcher, "Prince Moonlight", T'oung Pao LXVIII, 1982, p. 24, 45; et Carmen Blackcr, "Thc Divinc Boy in Japancsc Buddhism", Asian Folklore Studies XXII, 1963, p. 77-88.

43) Cf. H. Durt, “La 'visite aux laboureurs' et la 'méditation sous l'arbre Jambu' dans les biographies sanskrites et chinoises du Buddha”, Indological and Buddhist Studies in Honour of Prof. J.W. de Jong, Canberra, 1982, pp. 110-111.

44) Rouleau peint, daté de 1254, par Keinin 度忍 et Shōjumaru 聖聚丸, $1^{\mathrm{C}}$ partie au Musée Nezu 根津, Tōkyō; $2^{\mathrm{e}}$ partic au Musée Gotō 五島, Tōkyō. Cf. E-ingakyō 給因果称, Nihon Emakimono zenshu 日本粭然物全集, vol. I, Kadokawa 角川, Tōkyō, 1977, pp. 58-62.

45) Gangðði Bunkazai kenkyūsho 元興寺文化財研究所, Bukkvō hōe no chōsa-Nehan- $\varepsilon$ chōsa hökokusho 佛教法合の调查・涅柴合調在報告急, Nara, 1978, fig. 30-34. 